\title{
Trivium
}

Revue franco-allemande de sciences humaines et sociales - Deutsch-französische Zeitschrift für Geistesund Sozialwissenschaften

$3 \mid 2009$

Droits subjectifs et droits de l'homme

\section{Droits subjectifs et dignité humaine. En guise d'introduction}

\section{Christoph Menke}

Traducteur : Olivier Mannoni

\section{(2) OpenEdition}

Journals

Édition électronique

URL : http://journals.openedition.org/trivium/3309

DOI : $10.4000 /$ trivium.3309

ISSN : 1963-1820

Éditeur

Les éditions de la Maison des sciences de l'Homme

Référence électronique

Christoph Menke, « Droits subjectifs et dignité humaine. En guise d'introduction », Trivium [En ligne], 3 । 2009, mis en ligne le 15 avril 2009, consulté le 22 septembre 2020. URL : http://

journals.openedition.org/trivium/3309; DOI : https://doi.org/10.4000/trivium.3309

Ce document a été généré automatiquement le 22 septembre 2020.

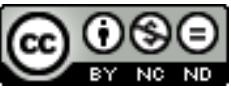

Les contenus des la revue Trivium sont mis à disposition selon les termes de la Licence Creative Commons Attribution - Pas d'Utilisation Commerciale - Pas de Modification 4.0 International. 


\title{
Droits subjectifs et dignité humaine. En guise d'introduction
}

\author{
Christoph Menke \\ Traduction : Olivier Mannoni
}

\section{Deux lectures des droits subjectifs}

1 Dans les études qu'il a consacrées au droit des sociétés modernes, Max Weber a interprété la figure des droits subjectifs comme le résultat d'une mutation fondamentale dans la compréhension du "droit légitime» (Berechtigung). Le droit traditionnel fixe des devoirs à l'individu, lui dit ce qu'il doit faire et le menace des conséquences qu'entraînerait le fait de s'en abstenir. Un tel ordonnancement des devoirs engendre à son verso - « sous la forme d'un 〈reflet $\iota^{1} »-$ des droits légitimes. Cela vaut au sens élémentaire où le fait de fixer et d'imposer les cadres juridiques du devoir, par exemple celui de ne pas voler, produit de l'autre côté le droit légitime de ne pas être volé. Mais il n'en découle pas pour l'autre un «droit subjectif » de ne pas être volé. Seul le droit moderne parle d'un droit à la propriété (ou même à la vie, à la liberté, etc.) - et retourne ainsi la perspective : Le droit légitime de l'un n'est plus le «reflet » $\mathrm{du}$ devoir des autres, mais produit au contraire ce devoir. Le droit légitime de l'individu devient une capacité, une "source de pouvoir " (Weber). Il s'agit là de l'acquisition du droit légitime au sens de ce que l'on appelle les « droits subjectifs » : les droits subjectifs confèrent un pouvoir : ils donnent à l'individu un pouvoir sur les actes des autres.

Dans son étude du droit moderne, Weber a aussi expliqué pourquoi a été introduite cette nouvelle figure du droit subjectif. Weber cite deux facteurs différents dont la coexistence tendue se situe à l'arrière-plan des oppositions qui marquent jusqu'à ce jour les débats sur les droits subjectifs : "Les forces décisives quant à ce changement dans les formes techniques de la création autonome du droit étaient d'une part, au point de vue politique, les besoins de puissance ressentis par les souverains et fonctionnaires d'une l'institution étatique qui allait en se confortant, d'autre part, au point de vue économique, les intérêts des participants au marché [...] c'est-à-dire ceux 
qui sont privilégiés dans la lutte formellement libre > pour la concurrence et les prix sur le marché, en vertu de leur position sociale en tant que propriétaires ( « situation de classe $>»^{2}$. Ce sont donc deux facteurs qui font émergerla nouvelle forme des droits subjectifs. Le premier facteur est une transformation fondamentale de l'ordre politique: les nombreux ordres intermédiaires des sociétés féodales sont battus en brèche par la naissance de l'État moderne qui monopolise la Gewalt, le pouvoir et la force, et se trouve ainsi directement confronté à tous ses citoyens. Les droits subjectifs se rapportent à cette relation entre le citoyen et l'État qui définit l'ordre politique moderne. L'autre facteur est la naissance d'une sphère sociale libérée des régulations politiques normatives. Le droit devient ainsi l'instance qui protège et garantit tous les accords que les membres de la société civile ont conclus «formellement, librement ${ }^{3}$ ». Les droits subjectifs leur donnent des "compétences ${ }^{4}$ », la garantie que leurs contrats sont protégés par le droit.

Du fait de la double fonction qu'ils remplissent dans les sociétés modernes, la figure des droits subjectifs se trouve traversée par une ambivalence qui est restée déterminante jusque dans les débats actuels. On le voit de manière exemplaire dans la tension non résolue qui affecte la compréhension de la liberté cautionnée par le droit. Chez Kant, déjà, l'idée de l'autonomie rationnelle et le concept de volonté privée entrent durement en conflit ${ }^{5}$. Cette tension se reflète dans deux interprétations tout à fait différentes de la liberté garantie par les droits subjectifs :

D'un côté, on trouve la thèse, soutenue de manière exemplaire par Amartya Sen, selon laquelle le sens du pouvoir conféré par les droits subjectifs consiste à «donner la capacité » à des sujets de participer à des pratiques sociales ${ }^{6}$. La liberté cautionnée par le droit est la liberté en tant que faculté subjective, et les facultés subjectives sont celles qui permettent l'exercice des pratiques sociales, la participation sociale. Sen propose une reformulation sociale du concept d'autonomie et considère que le sens des droits est d'en ouvrir la possibilité. Sen tire ainsi la conséquence de la rupture avec l'idée traditionnellement libérale de la protection de la « liberté négative ${ }^{7}$ » qu'a entreprise le libéralisme actuel aux États-Unis - Ronald Dworkin, John Rawls, ou sous une autre forme Joseph Raz: lorsque l'idée des droits subjectifs est libérée des fausses idéologies du sujet, celui-ci n'est qu'un vecteur de l'acquisition sociale de la capacité à être autonome.

De l'autre côté, on trouve la thèse selon laquelle la forme des droits subjectifs implique qu'ils exposent des titres de droit dont la revendication revient à la volonté de l'individu. Avoir des droits subjectifs signifie seulement la possibilité d'y prétendre; dans cette mesure, le devoir des autres, qui leur correspond, est à l'appréciation du détenteur du droit ${ }^{8}$. Dans son analyse de la figure du droit subjectif, Niklas Luhmann a formulé ce point décisif en ces termes : « Il [c'est-à-dire le droit] ne veut vouloir que si et dans la mesure où l'individu veut, et ne pas vouloir que l'individu ne veut pas; il reprend la volonté de l'individu à l'aune de la volonté privée9. " La liberté qu'ouvrent les droits subjectifs n'est par conséquent pas entendue ici comme la capacité de participer à la société, mais comme l'ouverture de champs d'action de la volonté privée $^{10}$. La forme des droits subjectifs, quel que soit leur contenu, représente la capacité conférée à l'individu dans son arbitre libéré de détermination normative. 


\section{Droits subjectifs et dignité humaine}

6 On trouve ainsi, dans un rapport d'opposition tendue, deux définitions du sujet des droits: le sujet comme membre social et le sujet comme individu privé. Cette opposition dans l'interprétation de la forme des droits subjectifs se répète dans la manière dont le rapport entre cette forme et la teneur normative des droits de l'homme est défini. On peut dire, dans un premier temps, que les droits de l'homme sont une catégorie déterminée des droits subjectifs, celle des droits fondamentaux : les droits dans lesquels il est question du statut du sujet de droit en tant que tel. Ces droits sont en règle générale compris dans trois catégories : les droits libéraux à la protection, les droits démocratiques à la participation et les droits sociaux aux prestations. Une première question est de savoir si la simple forme des droits subjectifs porte déjà en elle ces contenus normatifs ambitieux. Si l'on répond à cette question par l'affirmative, on peut soutenir que l'on se trouve face à une contradiction lorsque des États comme les dictatures des pays asiatiques en développement, par exemple, introduisent un ordre de propriété capitaliste sans droits fondamentaux ${ }^{11}$.

7 La question des droits de l'homme ne porte cependant pas seulement sur le contenu des droits subjectifs fondamentaux, mais aussi sur leurs détenteurs; il ne s'agit donc pas seulement de savoir quels droits sont en vigueur (ou doivent l'être), mais de savoir qui a ou doit avoir de tels droits fondamentaux. Dans le débat allemand, cette question est le plus souvent traitée sous l'intitulé de la "dignité humaine». On se réfère alors à l'article 1 de la Loi fondamentale allemande de 1949: «La dignité de l'homme est intangible. La respecter et la protéger est le devoir de tout pouvoir d'État. » La Loi fondamentale reprend donc à son compte le lien que la Déclaration universelle des Droits de l'homme de 1948 établit explicitement, dans son préambule, entre l'appel à la dignité humaine et l'expérience des «actes de barbarie » totalitaires ${ }^{12}$. Le concept de dignité humaine affirme ainsi que chaque homme est digne d'être reconnu comme un détenteur de droits subjectifs.

Dans la philosophie et les sciences juridiques allemandes, l'opinion dominante a longtemps été que le concept de dignité humaine désigne une "valeur » normative suprême qui est prédonnée à l'ordre politique sans qu'il puisse en disposer ; ce point de vue a ensuite pu être formulé en termes thomistes ou kantiens ${ }^{13}$. On postule toujours ici un ordre de valeur pré-, voire supra-politique, que l'on peut appréhender dans la connaissance morale et face auquel la politique se retrouve dans le simple rôle d'une «application». Cette position a fait l'objet de critiques croissantes, pour des motifs tenant autant à la théorie de la rationalité qu'à la théorie politique (même si elle joue de nouveau un rôle dans les débats bioéthiques récents ${ }^{14}$ ). Que la "dignité humaine » ne puisse constituer un principe autonome permettant de déduire l'ordre de droit ne signifie cependant pas que le concept ait perdu sa signification pour la détermination des droits. Cette signification tient à la contribution qu'apporte le concept de dignité humaine à la clarification de la question formulée ci-dessus : qui est le détenteur des droits subjectifs et, par conséquent, comment doit être compris le sujet de ce type de droits?

9 Le concept de dignité répond à la question du sujet des droits dans la mesure où il désigne «l'homme » comme ce sujet. L'emphase de cette réponse tient au fait que tous (les hommes) sont des détenteurs de droits; que nul en conséquence ne doit être exclu de la subjectivité juridique. Mais cette emphase masque le fait que la référence à 
«l'homme» comme sujet des droits implique que l'on détermine un destinataire spécifique. Cela apparait le plus clairement dans les traquenards du naturalisme qui entourent le concept « d'homme ». Hegel a ainsi déjà formulé l'argument selon lequel la capacité d'avoir un droit ne peut pas se référer à «l'homme comme être naturel »; car c'est justement à l'homme naturel et à ses différentes capacités que se réfère la justification de l'esclavage, contre la thèse selon laquelle tous les hommes sont des sujets de droit ${ }^{15}$. Cette critique de la «naturalisation » qui réside dans le concept de l'homme a été reformulée avec force par Hannah Arendt ${ }^{16}$, et elle a indiqué à cette occasion comment une compréhension non naturaliste devrait comprendre la dignité de l'être humain : elle doit prendre l'être humain comme un être non pas naturel, mais essentiellement «politique » (Arendt) ou « spirituel » (Hegel). L'homme a une dignité et par là-même une capacité d'avoir des droits dans la mesure où sa destination est d'être membre d'une société. C'est son « droit aux droits ${ }^{17}$.

Cette réponse, apportée par le concept de dignité humaine à la question du sujet des droits, correspond à la première des deux définitions des droits subjectifs citées plus haut: celle des droits comme ouverture d'une possibilité de participation sociale. Mais ce qui constitue la dialectique de la "dignité humaine ", c'est le fait que le concept de l'homme représente aussi le principe de la lecture opposée, individualiste, des droits subjectifs. Dans cette deuxième lecture, le concept de dignité humaine signifie que l'homme ne se réduit pas à sa définition " politique » ou "spirituelle » de membre de la société. Même s'il manque de clarté dans son articulation, même s'il est insuffisamment fondé, il constitue l'autre face de l'héritage antitotalitaire du concept de dignité humaine ${ }^{18}$. «L'homme » y symbolise « l'autre face » (Luhmann) dans le sujet, face qui ne peut être totalement intégrée sur le plan social. La dignité de l'homme, dans cette deuxième lecture, tient à la prétention justifiée qu'a l'homme de rester en dehors de la société, et la forme des droits subjectifs tire précisément sa justification de ce qu'elle fait valoir cette prétention de la même manière pour tous en donnant un champ d'action à la volontéprivée de l'individu, non régulable par des normes.

\section{Le paradoxe de l'homme et la dialectique des droits}

11 Les positions visant à légitimer le droit tranchent le plus souvent en faveur de l'une des deux lectures des droits subjectifs et décrivent les droits comme une manière de rendre possible ou bien l'intégration à une société, ou bien la volontéindividuelle. En revanche, les positions critiques à l'égard du droit, qui prennent leurs racines dans le communautarisme ${ }^{19}$, dans le marxisme ${ }^{20}$ ou dans les deux ${ }^{21}$, soulignent l'intrication «dialectique » de l'intention des droits subjectifs qui est de conférer une capacité sociale avec la libération de fait qu'ils offrent à la volonté privée. Ils le font dans l'intention critique de mettre au jour la libérationdes rapports sociaux de domination qui est liée à la libération juridique de la volonté privée (si même ce n'est pas son véritable objectif) : dans la mesure où les droits subjectifs libèrent la volonté privée de tout contrôle normatif, ils soustraient simultanément à la critique les rapports sociaux de domination. Mais cette association dialectique de la liberté et de la domination vaut aussi pour l'autre face des droits subjectifs : rendre possible l'appartenance sociale, c'est aussi toujours à la fois conférer des capacités et par conséquent libérer, et normer et par conséquent discipliner. On peut y lire comme un indice du fait que c'est précisément la relation dialectique des différents aspects qui constitue la forme des 
droits subjectifs. C'est en cela que réside la valeur ou l'apport de cette forme pour les sociétés modernes : en elle, la liberté et la domination sont indissociablement liées l'une à l'autre, et ce parce que l'une et l'autre sont pertinentes pour la forme des droits subjectifs, dans les deux lectures, que l'on considère que les droits subjectifs sont ce qui rend possible l'intégration sociale ou qu'ils sont ce qui libère la volonté individuelle. Une analyse du concept de dignité humaine peut contribuer à faire comprendre pourquoi cette tension constitutive du droit est insoluble. La raison en est la figure paradoxale de l'homme comme détenteur des droits.

\section{BIBLIOGRAPHIE}

Adorno, T.W. (1997) : «Aldous Huxley und die Utopie », in : Gesammelte Schriften, vol. 10, Francfort-sur-le-Main, Suhrkamp.

Agamben, G. (2001) : «Jenseits der Menschenrechte », in : id. : Mittel ohne Zweck, Fribourg / Berlin, Diaphanes, p. 23-34.

Alexy, R. (1994) : Theorie der Grundrechte, Francfort-sur-le-Main, Suhrkamp.

Arendt, H. (1949) : « « The Rights of Man `. What Are They? », in : Modern Review, vol. 3, n 1, p. 24-36.

Berlin, I. (1969) : Four Essays on Liberty, Oxford, Oxford University Press.

Böckenförde, E.-W. / Spaemann, R. (éd.) (1987) : Menschenrechte und Menschenwürde. Historische Voraussetzungen - säkulare Gestalt - christliches Verständnis, Stuttgart, Klett-Cotta.

Brown, W. (2002) : "Suffering the Paradoxes of Rights ", in : Brown, W. / Halley, J. (éd.) : Left

Legalism / Left Critique, Durham / Londres, Duke University Press, p. 420-433.

Dürig, G. (1956) : « Der Grundrechtssatz von der Menschenwürde », in : Archiv des öffentlichen

Rechts, cahier 2, p. 117-157.

Enders, C. (1997) : Die Menschenwürde in der Verfassungsordnung. Zur Dogmatik des Art. 1 GG, Tübingen, Mohr (Siebeck).

Feinberg, J. (1980) : Rights, Justice, and the Bounds of Liberty, Princeton, Princeton University Press.

Glenden, M.A. (1991) : Rights Talk, New York, The Free Press.

Geuss, R. (2005) : Outside Ethics, Princeton, Princeton University Press.

Habermas, J. (1992) : Faktizität und Geltung. Beiträge zur Diskurstheorie des Rechts und des demokratischen Rechtsstaats, Francfort-sur-le-Main, Suhrkamp, 1992.

Habermas, J. (1999) : « Zur Legitimation durch Menschenrechte », in : Brunkhorst, H. / Niesen, P. (éd.) : Das Recht der Republik, Francfort-sur-le-Main, Suhrkamp, p. 386-403.

Haucke, K. (2003) : Das liberale Ethos der Würde, Wurtzbourg, Königshausen \& Neumann.

Hegel, G.W.F. (1970) : Grundlinien der Philosophie des Rechts, Francfort-sur-le-Main, Suhrkamp.

Höffe, O. (2002) : Medizin ohne Ethik?, Francfort-sur-le-Main, Suhrkamp. 
Honneth, A. (1992) : Kampf um Anerkennung. Zur moralischen Grammatik sozialer Konflikte, Francfortsur-le-Main, Suhrkamp.

Kelsen, H. (1960) : Reine Rechtslehre, Vienne.

Luhmann, N. (1965) : Grundrechte als Institution, Berlin, Dunker \& Humblot.

Luhmann, N. (1981) : « Subjektive Rechte: Zum Umbau des Rechtsbewusstseins für die moderne Gesellschaft ", in : id. : Gesellschaftsstruktur und Semantik, vol. 2, Francfort-sur-le-Main, Suhrkamp, p. 45-104.

Luhmann, N. (2005) : « Die Tücke des Subjekts und die Frage nach dem Menschen », in : Soziologische Aufklärung, vol. 6, Wiesbaden, VS, p. 149-161.

MacIntyre, A. (1981) : After Virtue, Notre Dame, University of Notre Dame Press.

Marshall, T.H. (1964) : Class, Citizenship, and Social Development, Garden City/N.Y., Doubleday.

Menke, C. / Pollmann, A. (2007) : Philosophie der Menschenrechte. Zur Einführung, Hambourg, Junius.

Sen, A. (1992) : Inequality Reexamined, New York / Oxford, Russell Sage Foundation et al.

Weber, M. (1980) : Wirtschaft und Gesellschaft. Grundriss der verstehenden Soziologie, éd. par Johannes Winkelmann, Tübingen, Mohr (Siebeck). Le passage cité est repris dans une édition partielle, du même (2007) : Sociologie du droit, traduit et introduit par Jacques Grosclaude, Paris, PUF.

Wellmer, A. (1993) : « Freiheitsmodelle in der modernen Welt », in : Endspiele: Die unversöhnliche Moderne, Francfort-sur-le-Main, Suhrkamp, p. 15-53.

Wetz, F.J. (2005) : Illusion Menschenwürde. Aufstieg und Fall eines Grundwerts, Stuttgart, Klett-Cotta.

Zimmermann, R. (2005) : Philosophie nach Auschwitz. Eine Neubestimmung von Moral in Politik und Gesellschaft, Reinbek bei Hamburg, Rowohlt.

\section{NOTES}

1. Weber (1980), p. 398 ; cf. Kelsen (1960), chap. 29.

2. Weber (1980), p. 419. Traduction in Weber (2007), p. 79 (traduction modifiée - O.M.).

3. Weber, avec Marx.

4. Alexy (1994), p. 211 sqq.

5. Habermas (1992), p. 142 sq.

6. Sen (1992); cf. Honneth (1992), chap. 5, se rattachant à Marshall (1964).

7. Berlin (1969); cf. Geuss (2005).

8. Feinberg (1980), p. 156 sqq.

9. Luhmann (1981), p. 66.

10. Cf. Wellmer (1993).

11. Par exemple Habermas (1999).

12. Menke / Pollmann (2005); Wetz (2005); Zimmermann (2005).

13. Cf. Böckenförde / Spaemann (1987) ; Dürig (1956).

14. Cf. Höffe (2002).

15. Hegel (1970), § 57.

16. Arendt (1949); cf. Agamben (2001).

17. Cf. Enders (1997).

18. Ce motif se trouve chez des auteurs aussi différents qu'Adorno (1951), Plessner (cf. Haucke [2003]) et Luhmann (2005). 
19. Glenden (1991).

20. Brown (2002).

21. MacIntyre (1981)

INDEX

Mots-clés : droits de l'homme, droits (subjectifs), citoyenneté, dignité humaine

Schlüsselwörter : Menschenrechte, Rechte (subjektive), Staatsbürgerschaft, Menschenwürde

\section{AUTEURS}

\section{CHRISTOPH MENKE}

Christoph Menke est professeur de philosophie à l'Université de Potsdam. Pour plus d'informations, voir la notice suivante. 